\title{
Empathy-Motivated Forgiveness: The Influence of Empathy, Prior Experience, and Contextual Factors in Route to Forgiveness of a Transgressor
}

Patrick J. Aragon, Psy. D.

School of Psychology, Florida Institute of Technology, 150 W University Blvd, Melbourne, FL 32901, United States

\section{Abstract}

Is the process of forgiveness contingent upon the empathic response towards another person? It is suspected, that a victim cannot begin to act, and then forgive a transgressor before he or she is able to empathize with the transgressor's guilt and distress. The empathy-altruism hypothesis is one example that further exemplifies the motivation and connection of the empathic response with forgiveness. In interpersonal relationships, conflicts are inevitable. The motivation to mend these problems and reconcile with the offender occurs through the experience of empathy and the altruism to act with positive regard. Prior experience of a situation is not a necessity for empathy-motivated forgiveness to occur. Fifty women and 20 men participated in this study (mean age of the respondents was 25.31). Participants answered questions from two vignettes and three scales, the New Empathy Scale the Forgiveness Scale, and the Forgiveness Likelihood Scale to assess the relationship between empathy and forgiveness, as well as the effect of prior experience. Empathy was correlated with forgiveness, according to the Forgiveness Likelihood Scale but not the Forgiveness Scale. Empathic individuals significantly forgave a transgressor without previously experiencing a similar situation. Religiosity was also correlated with all three scales. Findings extend prior research by demonstrating that empathy is a possible motivator of conciliatory behavior. Considerations of additional influences of empathy-related forgiveness were considered, and a pattern of gender on both empathy and forgiveness was also considered.

\section{Introduction}

\section{Empathy-motivated forgiveness}

Empathy is not a recent concept of interest in psychology, but its effect on forgiveness has sparked new consideration. Empathy is "the intellectual identification with or vicarious experiencing of the feelings, thoughts, or attitudes of another" (www.dictionary.com) Empathy is also defined as the desire to increase another person's personal welfare before consideration of one's own [1-3]. It is believed that Forgiveness of another can occur because of an empathic response to the other person. The purpose of this study, therefore, is to examine the influence of empathy on the process of forgiveness. Numerous variables contribute to forgiveness, but the focus of this review is on the role of empathy in the forgiveness process.

When one is hurt or offended, several variables affect one's reaction: a) whether the offender is a stranger, a friend, or a loved one; b) the strength of the relationship; c) the severity of the offense; and d) the previous experience of a similar transgression [4]. Thus, forgiveness is affected by contextual and person-specific factors. Both the offending partner and the offended partner can influence the likelihood that forgiveness will be achieved [5,6]. The empathic response of an offended person in relation to a transgression may be pivotal in the overall process of forgiveness. Empathy is also considered relevant (and sometimes vital)in the ability to continue to forgive, after the initial resolution [7].

\section{Forgiveness}

Trait forgiveness is the capacity to forgive interpersonal transgressions across situations over time. It is very often reliant on a pattern of contemplation, as emotion peaks immediately following the transgression, and slowly declines as time passes [8]. Dispositional forgiveness, by contrast, is an enduring personality trait; it is not concerned with the situation or transgressor. This type of [9]. United States; E-mail: paragon@fit.edu org/10.15344/2455-3867/2016/114 and source are credited.

\section{Publication History:}

Received: March 01, 2016

Accepted: June 01, 2016

Published: June 03, 2016

\section{Keywords:}

Empathic process, Altruism, Transgressions-against, Conflict specific forgiveness is the forgiveness of a specific person for a single transgression. Dyadic forgiveness is forgiveness in the context of a relationship with a specific person and occurs over the history of transgressions. All these types of forgiveness describe a process, and are not just a goal or intended end result.

Sometimes forgiveness may occur as a negative process. For instance, motives are negative when an individual forgives another: to a) manipulate that person, b) to make that person feel indebted, or c) to create guilty feelings in order to punish that person. Another negative process involves delay: i.e., an individual has already forgiven the offender, but does not communicate it to the offender; this is known as silent forgiveness, which prevents recovery for the offender. Conversely, one may communicate forgiveness to the offender even without experiencing it internally; this is known as hollow forgiveness

Positive processes in forgiveness include emotional and interpersonal forgiveness. Emotional forgiveness is the process of replacing the negative emotions associated with the failure to forgive with positive emotions, such as sympathy, empathy, and love. Similar to this is interpersonal forgiveness, in which an individual in an intimate relationship forgives the transgressions of a partner because he or she empathizes with the other's distress or guilty feelings.

"Corresponding Author: Patrick J Aragon, Psy. D., School of Psychology, Florida Institute of Technology, 150 W University Blvd, Melbourne, FL 32901,

Citation: Aragon PJ (2016) Empathy-Motivated Forgiveness: The Influence of Empathy, Prior Experience, and Contextual Factors in Route to Forgiveness of a Transgressor. Int J Psychol Behav Anal 2: 114. doi: http://dx.doi.

Copyright: (c) 2016 Aragon. This is an open-access article distributed under the terms of the Creative Commons Attribution License, which permits unrestricted use, distribution, and reproduction in any medium, provided the original author 
Forgiveness should also be distinguished from: forgetting, condoning, excusing, or denying that the offense has ever happened [10]. These actions are often described as the ineffective way to deal with a transgression.

There are a number of different explanations for how one forgives another. Some therapists consider forgiveness as the function of responsibility attribution. Others consider the relationship commitment itself a primary factor in the process of forgiveness. The quantifiable view of forgiveness is that it is a series of steps that people must complete, which are essential in the reduction of a wide range of psychological and physiological symptoms that occur when forgiveness has not been reached. Empathy for the transgressor is another emphasis in the research on the process of forgiveness [11,12].

\section{Empathy}

Empathy is a widely studied construct. It has been considered from the perspective of the emotional connection one feels to psychological objects, as in the connection between a mother and her child. It also has been considered the primary tool utilized by psychotherapists. Empathy originated from the term Einfuhlung, which in German means "the projection of the self into the object of beauty" [12] Einfuhlung was actually first incorrectly translated as sympathy. The confusion between the terms sympathy and empathy, however, is common, and is considered by some to overshadow the deeper, more complex, process of empathy. Empathy, by contrast, is the tendency for observers to project themselves into the objects of their perception, a kind of animism. This refers to the process whereby one person tries to understand accurately, emotionally and without prejudice, the subjectivity of another person. Sympathy is viewing the emotional experience of another from the outside, whereas empathy is viewing the emotional experience of another by taking in those emotions and experiencing them from the inside.

\section{Empathy-altruism hypothesis}

Much of the research on empathy relates it to the concept of altruism. Whereas empathy is the internal connection with the individual in need, altruism is the prosocial motivation to help that individual $[13,14]$. The empathy-altruism hypothesis argues that empathy encourages altruistic behavior: an individual helps another individual, or considers the other person's welfare first, strictly based on altruistic reasons, and does it with no concern or anticipation of reciprocation $[1,1$.

Altruism has been difficult to explain. Why does one do an unnecessary act if no positive outcome to the actor will occur if it is completed, and no negative outcome will occur if it is not completed? The empathy-altruism hypothesis suggests that one's empathic reaction to a person in need mediates the altruistic response [16].

To increase another person's welfare before one's own is thus, a matter of a true desire to help another based on the occurrence of empathy experienced for the individual in need. For example, a truly altruistic experience would be the escorting of an older adult across the street, or holding the door when someone's hands are full. It is best expressed when the action to help is described as "acting without thinking" [16]. To think about helping implies the need for a reason to complete the task, and that altruistic motivation is not enough.

Krebs [16] found that participants who experienced the strongest empathic reactions towards another individual were most willing to help that individual, regardless of their own welfare or loss of reward. The study paired two individuals, a participant and a confederate. Between the two individuals was a roulette wheel. The instructions were given that when the confederate landed on an even number, he or she would win money. Conversely, when he or she landed on an odd number he or she would receive an electric shock. The participant was led to believe that the position of the performer and the observer was decided randomly. After a few trials, the participants were told they would be given a bonus spin, in which they could win between 0 and $\$ 2$, depending on how much they wagered. If the ball landed on an even number, they would win the amount they wagered. However, if the ball landed on an odd number, the performer (the confederate) would receive a shock relative to the amount wagered by the participant. The results of the study revealed the reward was more than irrelevant, and the concern to help the other individual was more important. Additionally, the strongest empathic reactions in the participants led to the greatest concern, and therefore, altruistic behavior towards the confederate in the form of harm prevention (i.e., shielding him or her from the electric shock).

\section{Empathy and forgiveness}

Developing empathy is a necessary step in forgiveness. Having greater trait empathy makes it easier to forgive than someone having no trait empathy. Offense-specific and dyadic forgiveness are the primary types more often associated as important with forgiveness. A different mentality is involved when empathy is developed for a stranger. An individual must create a previously nonexistent connection with a stranger in order to be able to forgive him or her. Interpersonal forgiveness, however, is more complex than the altruistic helping of strangers [17].

Although the empathy-altruism hypothesis has been supported in cases of helping, is it also relevant in forgiveness? Consider the situation in reverse: now this victim (the offender) is trying to alleviate his or her distress by being forgiven. First, some situation has occurred in which the offended has begun to avoid or estrange the offender. The offended then witnesses the distress of the offender. The offended, ideally, empathizes with the offender (considers what it would be like to be the offender and be in distress) and is altruistically motivated to help alleviate the distress. Forgiveness, then, is a way to reduce the victim's need, or alleviate the distress of the offender [12].

It is possible to consider the relationship of empathy, forgiving, and the behaviors that follow as similar to what happens when empathy leads to the motivation to act altruistically towards another. The empathic connection necessary to help another in need is similar to the empathic connection to forgive another who has transgressed. Positive attachment and a shared history often will increase both empathy for the individual as well as the potential for forgiveness.

In general, an existing interpersonal relationship is based on a shared agreement of well-being for one another. However, a destructive occurrence, either one that is harmful or offensive to one of the partners, can upset the balance of well-being. The process of interpersonal forgiveness does involve variables other than that of empathy. This includes but is not limited to social-cognitive aspects, offense level of the action, relationship level, and personality type. However, all of the above are interconnected with the effects of empathy [6].

The conciliatory motivation, characterized here as forgiveness, is much the same as the empathic motivation to altruistically help or 
care for others. In addition, an apology by the offending partner facilitates increased empathy for the offending partner, possibly alerting the offended partner to the distress and guilt being experienced [18]. This does not imply immediate forgiveness, but instead may be the possible beginning of the process of forgiveness. This process of forgiving is the empathy-motivated set of motivational changes, in which the offended partner is more inclined towards prosocial actions in relation to the offending partner [12]. However, forgiving is not motivation in of itself, it simply is the term applied to the transformation of decreasing motivation to retaliate and estrange from the offending partner, and increasing conciliatory behavior as a result of the empathic response. This is an important concept to keep in mind when considering the behavioral response of forgiving another, and with the review of the upcoming study.

\section{Rationale for the study}

Forgiveness is the act of excusing a mistake or an offense by another, and it is initiated principally by emotion. However, cognitive processes also are involved in the assessment of the transgression experience. The primary emotional experience necessary for the initiation of forgiveness may be the experience of empathy. Conversely, the cognitive influence may be the reasoning to consider forgiveness, and thus unconsciously experience empathy. An empathic response towards a transgressor greatly increases the likelihood of forgiveness [12]. The lack of an empathic response should be effective in the prevention of forgiveness. Just as empathy is important in initiating forgiveness, the low occurrence of empathy should inhibit forgiveness from occurring.

Empathy is the emotional experience of relating to another person through which another's persons perceptions are cognitively taken as one's own. In order to alleviate the punishment of a person who has wronged another, all aspects must be taken into account. Included in this is the degree of negative emotional affect being experienced by the transgressor. If the offended empathizes with the offender, then the offended will understand and indirectly experience the negative emotional affect. With time, so that the empathic response can be evoked, experienced, and understood, the offended may feel a desire to relieve the need of the offender, by actively reducing the negative affect. Thus, conciliatory behaviors will then be initiated by the offended towards the offender; which is the process of forgiveness.

Either due to the nature of the offense or the ability to empathize, forgiveness may or may not occur. When the offense is too devastating, the offended person will be unable to empathize because of the lack of an emotional connection. In this case, an affective empathic response will not occur because no emotional connection is evoked and forgiveness is unlikely to be established. If a person does not empathize because he or she lacks the mental ability to empathize with the offender, then again no connection will be made. The result is that the negative affect will not be perceived and forgiveness is unlikely to be established [19].

Forgiveness of a transgressor is most apparent when the situation is familiar to the offended individual. The ability to form the necessary connection with someone, so that empathy will be experienced, occurs more frequently in a situation that is familiar, or has been experienced before. Thus, a person observing another in a situation that the person has experienced before should empathize more with the person in that situation. Experience may not be a prerequisite for empathy, but experience and familiarity can, it is suspected, increase an empathic response [20].
Consequently, a person of a high empathic nature, observing a situation that is similar to one experienced before, will experience a high empathy response to the person involved. If experience increases the empathic response, but is not required for it to happen, then it is suspected that the same individual will also experience a relatively high empathic response to a person even in a situation that has not directly been experienced before; however, not to same degree as the situation that was familiar to the individual. Therefore, the highly empathic individual will maintain a high level of empathy, and the familiar situation will only increase it. By contrast, a person of low empathic nature will be less inclined to empathize with another. In a situation similar to one previously experienced, the low empathic individual should empathize more than when in a situation not similar to one previously experienced, but not necessarily to a point adequate for forgiveness to occur. In this case, the level of empathic response remains low, but still should increase in a situation similar to one experienced before.

\section{Method}

\section{Participants}

Participants were 70 anonymous volunteers (50 women and 20 men), who participated online through the use of the following websites: www.socialpsychology.org/expts.html, and www.psych. hanover.edu/research/exponnet.html. The mean age of the respondents was 25.31 years $(\mathrm{SD}=4.09)$ with an age range of 18 to 36 years. Ethnicity was as follows: 44 (62.9\%) White participants, 18 (25.7\%) Hispanic participants, 4 (5.7\%) Asian/Pacific Islander participants, 3 (4.3\%) African-American participants, and one participant checked "other". Ninety-four percent reported at least one year of college experience, with the maximum number of years of college being eight years.

Of the sample, $68.6 \%$ reported a Christian religious affiliation, $24.3 \%$ reported a "nonreligious" religious affiliation, and the other $7.1 \%$ reported either an Islamic, Hindu, or Judaic religious affiliation. Career-field or college-major were distributed primarily through business, education, science, liberal arts, or human/health services (86\%).

\section{Materials}

Vignettes. Participants read two vignettes, both involving a person who is wronged in some way. Vignette 1 (the infidelity vignette):

A college student, T.J. feels overwhelmed with school. Also, T.J. was just dumped after a 3-year relationship. T.J. asked the ex what the reason was for the break up. The ex responded, "Maybe if you had put your book down and picked up the phone I might not have had to go out last week with someone else." Soon, T.J. found out through friends that the ex had been seeing someone else for a couple of weeks already. T.J. felt terrible, But T.J. saw during class and at other social gatherings that te ex felt very bad about hurting T.J.

After reading the vignette, participants responded to six items measuring empathic response and previous experience with the situation. Sample items include: "I feel sorry for T.J." and "I have had a friend who had an experience like the ex." The Likert-type response format ranged from 1 (Strongly disagree) to 5 (Strongly agree).

Vignette 2 (the journalism vignette): 
John is a journalist for the Enterprise Weekly. One day he is asked by a fellow journalist and friend for some help in getting an idea for a story. The man is desperate, and John sees that he is at risk of not making his deadline. John has been working on a story for the past few days, but obligated by the friend in need, he hands his story over to his friend. However, John had overestimated his ability to create a new story and misses his own deadline as a result. John's friend's story makes the front page, while John is told "he is not keeping up with company standards" and is let go. John's friend feels terrible about what happened.

Following vignette 2 were six statements measuring empathic response and previous experience with the situation. Sample items included: "I know from experience what it feels like to be John's friend" and "If I were John, I would eventually forgive his friend." The Likert-type response format ranged from 1 (Strongly disagree) to 5 (Strongly agree).

New Empathy Scale [21]. The New Empathy Scale consists of 29 statements and is designed to measure an individual's experience of empathy. Respondents were asked how well the statements describe their thoughts and feelings in particular situations. Sample items include "I feel other people's pain" and "If someone is upset I get upset, too." The Likert-type response format ranges from 1 (Strongly disagree) to 5 (Strongly agree). The New Empathy Scale contains six subscales, including Empathic Suffering (8 items), Positive Sharing (4 items), Responsive Crying ( 3 items), Emotional Attention (5 items), Feel for Others (4 items), and Emotional Contagion ( 2 items). The mean of the subscales reflects general empathy.

Cronbach's alpha for subscales of the New Empathy Scale were as follows: Empathic Suffering, .80, Positive Sharing, .71, Responsive Crying, .72, Emotional Attention, .63, Feel for Others, .59, and Emotional Contagion, .44. The Cronbach's alpha for the General Empathy Scale was 86 .

Forgiveness Scale [21]. The Forgiveness Scale measures forgiveness towards a particular offender. The scale consists of 15 items designed to assess affective and cognitive behavioral responses to transgressions. Respondents were asked to focus on the individual who mistreated or offended them, rather than a broad reaction to offenses in the past. Sample items include "I feel resentful towards the person who wronged me" and "I feel compassion for the person who wronged me." Additionally, the questions assess positive and negative responses to transgressions. The Likert-type response format ranged from 1 (Strongly disagree) to 5 (Strongly agree). Higher scores on the scale reflect greater forgiveness for the offender [21].

The Forgiveness Scale uses a two-factor solution, revealing adequate internal consistency and conceptually meaningful factors. The Absence of Negative factor (AN) subscale contains items describing the absence of negative feelings, judgments and behavioral-tendencies toward the transgressor. The Presence of Positive factor (PP) subscale contains items describing the presence of positive judgments, feelings, and behavioral-tendencies toward the transgressor. The Cronbach's alphas for AN were .86 , and for PP .85. The total reliability was .87 [21].

Forgiveness Likelihood Scale[21]. The Forgiveness Likelihood Scale measures the likelihood that an individual will forgive an offender in various situations. Respondents read 10 hypothetical scenarios and were asked to provide a meaningful judgment of each situation. The respondents were asked first to consider the scenarios as if they happened to them, and then respond to the likelihood of forgiving the offender in the situation. Sample items include "A stranger breaks into your house and steals a substantial sum of money from you. What is the likelihood that you would choose to forgive the stranger?" and "Your significant other has a 'one night stand' and becomes sexually involved with someone else. What is the likelihood that you would choose to forgive your significant other?" The Likert-type response format ranged from 1 (Not at all likely) to 5 (Extremely likely). Higher scores reflect a greater likelihood of forgiveness for the hypothetical offender. Cronbach's alpha for the scale was .85 [21].

\section{Procedure}

Participants completed the vignettes, the New Empathy Scale, the Forgiveness Scale, and the Forgiveness Likelihood Scale. The tests were not timed and all testing was done online. Included in the online survey was a brief demographics questionnaire completed after the above scales.

\section{Hypotheses and Analyses}

H1: The New Empathy Scale would correlate positively with the Forgiveness Scale and the Forgiveness Likelihood Scale.

H2: Participants receiving a high score of empathy, according to the New Empathy Scale, would forgive the transgressor, even though the situation is unfamiliar (by using selected questions from the infidelity vignette and the journalism vignette).

H3: Although experience is not a prerequisite, experience of a situation was expected to increase the empathic response, according to the New Empathy Scale. The participants will be more able to forgive a transgressor and empathize at a higher level, based on the situation being familiar (by using selected questions from the infidelity vignette and the journalism vignette).

H4: Religiosity would increase the participants' ability to empathize as well as forgive a transgressor, based on the New Empathy Scale, Forgiveness Scale, and Forgiveness Likelihood Scale.

Analysis of $H 1$ was a regression analysis computed to determine if a high score on the New Empathy Scale would predict a high score of forgiveness based on the Forgiveness Scale and the Forgiveness Likelihood Scale. Analysis of $\mathrm{H} 2$ was a regression analysis computed to determine if a high score on the New Empathy Scale would predict forgiveness of the transgressor in the infidelity vignette ( question 3), and the journalism vignette (question 5), as determined by selecting those who found the vignettes unfamiliar. Analysis of $\mathrm{H} 3$ was first a regression analysis computed to determine if a high score on the New Empathy Scale would predict forgiveness of the transgressor in the infidelity vignette (question 3 ), and the journalism vignette (question 5 ), as determined by selecting those who found the vignettes familiar. Then, the means of the significant results were compared to determine if those participants who found the vignettes familiar (according to the selected questions) empathized at a higher rate (according to the New Empathy Scale) then those who found the vignettes unfamiliar. Analysis of $H 4$ was a regression analysis of the question "How religious do you consider yourself?" and the three scales: New Empathy Scale, Forgiveness Scale, and the Forgiveness Likelihood Scale.

\section{Results}

\section{Empathy and forgiveness correlation (H1)}

To check the overall relationship between empathy and forgiveness, a correlation among the participants scores on the New Empathy Scale 
$(M=3.68, S D=0.45))$, the Forgiveness Scale $(M=3.35, S D=0.56)$, and the Forgiveness Likelihood Scale $(M=2.35, S D=0.71)$ was conducted. Scores on the Forgiveness Scale and the Forgiveness Likelihood Scale were significantly correlated, $r=.22, p=.033$, one-tailed. Scores on the New Empathy Scale and the Forgiveness Likelihood Scale were significantly correlated, $r=.20, p=.048$, one-tailed. However, scores on the New Empathy Scale did not correlate with the Forgiveness Scale. High Empathy Individuals Will Forgive a Transgressor in Unfamiliar Situations (H2)

For the infidelity vignette, 56 participants replied that T.J.s experience had not happened to them. Data from these participants were used in a correlation analysis between the New Empathy Scale score and the response to the following question, "If I were T.J. I would eventually forgive the ex." This correlation was significant, $r=.40, p=$ .003 , two-tailed. For the journalism vignette, 65 participants replied that John's experience had not happened to them. Data from these participants were used in a correlation analysis between the New Empathy Scale score and the response to the following question, "If I were John I would eventually forgive his friend." This correlation was significant, $r=.32, p=.010$, two-tailed.

From the infidelity vignette, data from the same group of participants (those who found the situations unfamiliar) were further analyzed. Responses to, "While reading the scenario I imagined myself as T.J." were correlated with scores on the New Empathy Scale. The correlation was significant, $r(56)=.32, p=.016$, two-tailed. For the journalism vignette the correlation was not significant.

\section{Experience increases the Likelihood of Empathy (H3)}

Those who considered the situations familiar were to be included in this analysis. However, this analysis was not conducted because only 14 and 5 participants could be included for the infidelity vignette and the journalism vignette, respectively.

\section{Religiousness and the empathic response (H4)}

Responses to "How religious do you consider yourself?" were correlated with the New Empathy Scale scores and both forgiveness scale scores. A significant correlation was found with all three scales: the New Empathy Scale, $r(69)=.29, p=.009$, one-tailed; the Forgiveness Scale, $r(69)=.45, p<.001$, one-tailed; and the Forgiveness Likelihood Scale, $r(69)=.34, p=.002$, one-tailed.

\section{Gender}

An independent sample t-test was conducted with all three scales to assess differences between genders, presented in Figure 1. On average, men experienced significantly greater forgiveness, according to the Forgiveness Likelihood Scale $(M=2.65, S D=0.85)$, compared to women $(\mathrm{M}=2.23, \mathrm{SD}=0.62)$. This difference approached significance $t(27.62)=2.04, p=.052$, and it also represented a medium sized effect $r=.36$. Although women experienced greater empathy $(M=3.70, S D$ $=0.44)$, than did men $(M=3.61, S D=0.49)$, this difference was not significant, $t(68)=.80, p=.43$. Although men experienced greater forgiveness, according to the Forgiveness Scale $(M=3.36, S D=0.63)$, compared to women $(M=3.35, S D=0.54)$, the difference was not significant, $t(68)=.05, p=.96$.

\section{Discussion}

The primary hypothesis, which empathy correlates with the process of forgiveness, was partially supported. The significant correlation between the New Empathy Scale and the Forgiveness Likelihood Scale

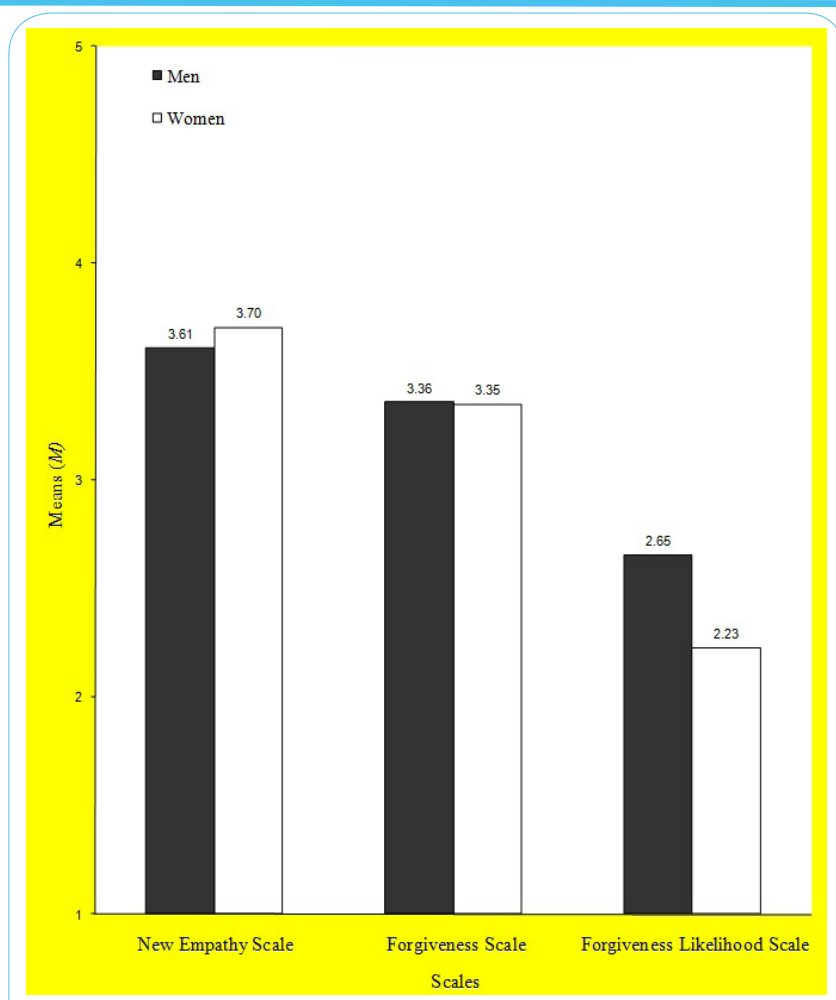

Figure 1: Gender differences in the New Empathy Scale, the Forgiveness Scale, and the Forgiveness Likelihood Scale.

suggests that people forgive others to the extent that they experience empathy for them. This supports the forgiveness hypothesis of McCullough et al. [12] in that once the empathic response of the offended partner overshadows the actions of the transgressor, a set of motivational changes begins and forgiveness can occur. The degree of empathy experienced by the participant, then, directly influences the possibility of experiencing forgiveness.

Scores on the Forgiveness Scale did not correlate with scores on the New Empathy Scale. The Forgiveness Scale was designed to assess affective and cognitive behavioral responses to transgressions [21]. Participants are asked to: "consider a person who has wronged or mistreated you in the past" and to consider this same (one) person for each of the subsequent statements. The Forgiveness Scale assesses primarily trait forgiveness, the capacity or ability to forgive an interpersonal transgression across situations over time [8]. The New Empathy Scale assesses an individual's "experience of empathy." An individual's capacity to forgive may not be parallel with his or her experience of empathy. Empathy is a personal experience, and is not necessarily congruent with the general capacity of forgiveness [22] assessed by the Forgiveness Scale. Perhaps an experience of empathy is not relevant in the process of forgiveness across situations, and over time, as the empathic experience is specific to the situation.

Participants' scores from both forgiveness scales correlated with each other. The Forgiveness Likelihood Scale was designed to assess the "likelihood" that a person will forgive another in a given context. This scale asks the participant to: "imagine the scenarios below happened to you....consider the likelihood that you would choose to forgive the person." The Forgiveness Scale asked the participant to consider "one person who has wronged you in the past, and consider that person for each statement." Even though the type of forgiveness is not the same for the scales, each scale measures an aspect of forgiveness. 
The Forgiveness Likelihood Scale assesses primarily offensespecific forgiveness, the forgiveness of a specific person for a single transgression, and the Forgiveness Scale assesses trait forgiveness. The distinction could have been the reason both for the relatively low correlation $(r=.22)$ between the scales and for the discrepancy related to the empathy correlations. Whereas the general concept of forgiveness is the process of ceasing to feel resentment or anger against another for a perceived offense or difference [9], empathy is personal and affected by individual circumstances. Thus, while both forgiveness scales correlated because each was measuring the similar construct of forgiveness, the New Empathy Scale only correlated with the Forgiveness Likelihood Scale because the scales were assessing individual experiences, rather than a more general consideration of the experience of empathy and forgiveness, respectively.

The second hypothesis was supported: empathic individuals will forgive transgressors in an unfamiliar situation. The significant correlation of the New Empathy Scale (for those selecting the vignette as unfamiliar) with the forgiveness question in each vignette, supported the idea that prior experience is not a necessity for empathy to occur $[20,23]$. Those participants who were found to be more empathic, from the New Empathy Scale, and were more likely to forgive (in both vignettes), even though the situation was unfamiliar. Some research suggests that prior experience may actually reduce the empathic response, if selfish motives are first associated with the experience [20]. Familiarity with the vignettes could have caused a reduction in the association of empathy to the transgressor, and may have resulted in fewer participants opting to forgive the transgressor.

The third hypothesis, that experience would increase the empathic response, was unable to be tested because participants did not find the vignettes familiar. This may have been caused by two things. First, it is possible that the question in reference to prior experience was too specific ("has T.J.s [John's] experience ever happened to you?"). The participant may have had similar experiences, but it is a limitation of the study that the question was asked so specifically, or that a second, more general, question was not asked. Perhaps the participants perceived the question too specifically, in that each and every aspect of the vignette had to be applicable to his or her prior experience for it to be considered familiar. For example, with the infidelity vignette, the participant first had an experience in which he or she had been broken up with, then discovered the partner had been unfaithful previously, and finally witnesses their ex-partner in distress about all that had transpired.

A second problem with the prior experience question may be that although gender was ambiguous in the infidelity vignette ("T.J" and "the ex"), the male character of the journalism vignette (John) may have limited the application of familiarity to a prior experience among the participants; specifically the female participants unable to successfully empathize with the male character.

The hypothesis that religiosity is related to empathy and forgiveness was supported, consistent with previous research [24,25]. One assumption, nonetheless, is that not all individuals in the process of forgiving look to religion, or wish to participate in a religiouslybased attempt, at forgiveness. Though, some religious individuals may appreciate the opportunity to participate in forgiveness interventions that explicitly address their own religious beliefs and practices [26] It could be argued, however, that religious forgiveness is forgiveness based on a principle or set of principles, and it may be different from the forgiveness offered by nonreligious individuals. Nonreligious forgiveness are conceived as following a set of steps or stages [9], whereas religious forgiveness may sometimes be "fast-tracked to a conclusion" based on the principles and guidelines instilled by that person's religious beliefs [27]. It remains a question whether religion encourages the teaching of forgiveness and its practice, or whether more forgiving individuals are drawn to religion. The nature of the transgression and negative feelings associated with the process of forgiveness may also be relevant to whether religion is considered in the process of forgiveness or not (e.g., attempting to forgive the person who murdered one's child, if the person's religious beliefs ask one to do so).

Unlike previous studies, men experienced significantly greater forgiveness than women (according to the Forgiveness Likelihood Scale; [8]. Conversely, neither the Forgiveness Scale nor the New Empathy Scale yielded a gender effect. One could argue that gender stereotypes, if they do predict a prominent effect, are more contextual. Additionally, although women generally have greater forgiveness than men, neither men nor women differ in their total capacity of forgiveness [17]. Whereas women may tend to be more forgiving or empathic in some conditions, it may not be the case across the board. For the New Empathy Scale, the circumstances were general, presenting a broad range of statements that could allow men and women to empathize equally (e.g., "Too much is made of the suffering of pets and animals" and "I would describe myself as a pretty soft-hearted person"). This was the same with the Forgiveness Scale (e.g., "I wish for good things to happen to the person who wrong me" and "I can't stop thinking about how I was wronged by this person"). The Forgiveness Likelihood Scale, however, is contextually based, formulated by a series of scenarios. Perhaps the nature of the scenarios was harder for the women participants to forgive than the men. Of the 10 scenarios, three were about the spreading of rumors, two about relationships, one about going to a dance, and another about the borrowing and loss of a personal item. Research has indicated that women may use indirect aggression more than men [28], and this assumption would suggest a higher rate of forgiveness among the male participants in this study based on the majority of the scenarios that were used.

Empathy has been regarded as a relatively high mental function among humans [22], whereas forgiveness at its core is a much simpler process. However, how is it then that empathy may be a requisite for an adult to process the experience of forgiveness? The present findings open a window to the investigation of this connection, and in what ways an empathic response may encourage forgiveness. Additionally, whereas the interaction between empathy and forgiveness has become relatively accepted, the influence of prior experience continues to be uncertain.

\section{Competing Interests}

The authors declare that they have no competing interests.

\section{References}

1. Batson CD, Batson JG, Griffitt CA, Barrientos S (1989) Negative state relief and the empathy-altruism hypothesis. Journal of Personality and Social Psychology 56: 922-933.

2. Cohen D, Strayer J (1996) Empathy in conduct-disordered and comparison youth. Developmental Psychology 32: 988-998.

3. Levenson RW, Ruef AM (1992) Empathy: A physiological substrate. J Pers Soc Psychol 63: 234-246. 
Citation: Aragon PJ (2016) Empathy-Motivated Forgiveness: The Influence of Empathy, Prior Experience, and Contextual Factorsin route to Forgiveness of a Transgressor. Int J Psychol Behav Anal 2: 114. doi: http://dx.doi.org/10.15344/2455-3867/2016/114

Page 7 of 7

4. Batson CD, Duncan BD, Ackerman P, Buckley T, Birch K (1981) Is empathic emotion a source of altruistic motivation? J Pers Soc Psychol 40: 290-302.

5. Cialdini RB, Schaller M, Houlihan D, Arps K, Fultz J (1987) Empathy-based helping: Is it selflessly or selfishly motivated? J Pers Soc Psychol 52: 749758

6. McCullough ME, Rachal KC, Sandage SJ, Worthington EL Jr, Brown SW et al. (1998) Interpersonal forgiving in close relationships: Il. Theoretical elaboration and measurement. J Pers Soc Psychol 75: 1586-1603.

7. Walker DF, Gorsuch RL (2004) Dimensions underlying sixteen models of forgiveness and reconciliation. Journal of Psychology and Theology 32: $12-25$.

8. Berry JW, Worthington EL, O'Connor LE, Parrott L, Wade NG (2005) Forgiveness, vengeful rumination, and affective traits. J Pers 73: 183-213.

9. Worthington EL Jr, Sharp CB, Lerner AJ, Sharp J (2006) Interpersonal forgiveness as an example of loving one's enemies. Journal of Psychology and Theology 34: 32-42.

10. Konstam V, Holmes W, Levine B (2003) Empathy, selfism, and coping as elements of the psychology of forgiveness: A preliminary study. Counseling and Values 47: 172-183.

11. McCullough ME, Root LM, Cohen AD (2006) Writing about the benefits of an interpersonal transgression facilitates forgiveness. J Consult Clin Psychol 74: 887-897.

12. McCullough ME, Worthington EL Jr, Rachal KC (1997) Interpersonal forgiving in close relationships. J Pers Soc Psychol 73: 321-336.

13. Weyl CF (1993) Empathy, perspective taking, and role taking: A review of the use of the constructs and an attempt at redefinition. Dissertation Abstracts International 54: 1117

14. Wispé $L$ (1986) The distinction between sympathy and empathy: To cal forth a concept, a word is needed. J Pers Soc Psychol 50: 314-321.

15. Batson CD, Ahmad N (2001) Empathy-induced altruism in a prisoner's dilemma II: What if the target of empathy has defected? European Journal of Social Psychology 31: 25-36.

16. Krebs D (1975) Empathy and altruism. J Pers Soc Psychol 32: 1134-1146.

17. Macaskill A, Maltzby J, Day L (2002) Forgiveness of self and others and emotional empathy. J Soc Psychol 142: 663-665.

18. Baumeister RF, Stillwell AM, Heatherton TF (1994) Guilt: An interpersonal approach. Psychological Bulletin 115: 243-267.

19. Regehr C, Goldberg G, Hughes J (2002) Exposure to human tragedy, empathy, and trauma in ambulance paramedics. Am J Orthopsychiatry 72 505-513.

20. Batson CD, Sympson SC, Decruz P, Todd MR, Weeks JL, et al. (1996) "I've been there, too": Effect on empathy of prior experience with a need. Pers Soc Psychol Bull 22: 474-482.

21. Rye MS, Loiacono DM, Folck CD, Olszewski BT, Heim TA, et al. (2001) Evaluation of the psychometric properties of two forgiveness scales. Current Psychology 20: 260-277.

22. Duan C, Hill CE (1996) The current state of empathy research. Journal of Counseling Psychology 43: 261-274.

23. Barnett MA, Tetreault PA, Masbad I (1987) Empathy with a rape victim The role of similarity and empathy: The experience of rape. Violence Vict 2: $255-262$.

24. Jose OA, Alfons $\vee(2007)$ Religiosity and forgiveness among first-married and remarried adults. Mental Health, Religion \& Culture 10: 379-394.

25. Van Dyke CJ, Elias MJ (2007) How forgiveness, purpose, and religiosity are related to the mental health and well-being of youth: A review of the literature. Mental Health, Religion, \& Culture 10: 395-415

26. Rye MS (2005) The religious path towards forgiveness. Mental Health Religion \&Culture 8: 205-215.

27. Block JH (1973) Conceptions of sex roles: Some cross-cultural and longitudinal perspectives. Am Psychol 28: 512-526. 